Cahiers $d u$ MONDE RUSSE

\section{Cahiers du monde russe}

Russie - Empire russe - Union soviétique et États indépendants

40/4 | 1999

Varia

\title{
Gilbert Romme à propos de l'armée russe au $\mathrm{XVIII}^{\mathrm{e}}$ siècle
}

\author{
Alexandre V. Tchoudinov
}

\section{OpenEdition}

Journals

Édition électronique

URL : https://journals.openedition.org/monderusse/28

DOI : $10.4000 /$ monderusse. 28

ISSN : $1777-5388$

Éditeur

Éditions de l'EHESS

Édition imprimée

Date de publication : 1 novembre 1999

Pagination : 723-750

ISBN : 2-7132-1341-X

ISSN : $1252-6576$

\section{Référence électronique}

Alexandre V. Tchoudinov, «Gilbert Romme à propos de l'armée russe au xvIII siècle », Cahiers du monde russe [En ligne], 40/4 | 1999, mis en ligne le 15 janvier 2007, consulté le 03 septembre 2022. URL : http://journals.openedition.org/monderusse/28; DOI : https://doi.org/10.4000/monderusse.28 


\section{GILBERT ROMME À PROPOS DE L'ARMÉE RUSSE AU XVIII' SIÈCLE**}

LES BIOGRAPHES DE GILBERT ROMME se sont souvent penchés sur l'histoire du séjour en Russie (1779/1786) de cet acteur en vue de la Révolution française ${ }^{1}$. Ils ont examiné les travaux de ce naturaliste que ses voyages ont mené de la Baltique à l'Oural, de la mer Blanche à la mer Noire et qui a collaboré avec presque tous les plus éminents spécialistes russes en sciences naturelles de son époque. Ses expériences dans le domaine de la pédagogie comme précepteur du jeune comte Paul Stroganov, que Romme éduquait et élevait en accord avec les théories de JeanJacques Rousseau, ont également retenu leur attention. Aussi s'est-il forgé en un peu plus d'un siècle dans la littérature historique l'image édifiante d'un Romme savant et pédagogue, remplissant avec abnégation une mission civilisatrice dans les rudes conditions d'un immense pays septentrional. Mais répandre les fruits des Lumières était-il le seul objectif de son séjour en Russie?

J'en ai douté pour la première fois en prenant connaissance de l'un des documents du fonds Romme conservés aux archives de la Section de Saint-Pétersbourg de l'Institut d'histoire russe, le mémoire intitulé Observations sur le Militaire de Russie en $1780^{2}$. Ce manuscrit de sept pages in-folio, contenu dans une chemise de papier jaune, n'est pas signé, mais que Romme en soit l'auteur est facile à établir

\footnotetext{
* La présente publication anticipe sur celle de l'ensemble de la Correspondance privée et des récits de voyage de Gilbert Romme, préparée par une équipe comprenant Philippe Bourdin, Jean Ehrard, Hélène Rol-Tanguy, Mihail Safonov et Alexandre Tchoudinov, pour paraître dans la collection Archives de 1'Est. Six volumes sont prévus.

Ce travail a bénéficié du soutien du Research Support Scheme de 1'Open Society Support Foundation, subvention 378/1998.

1. Cf., par exemple, M. de Vissac, Romme le Montagnard, Clermont-Ferrand, 1883 ; Gilbert Romme et son temps (1750-1795), Paris, 1966; A. Galante Garrone, Gilbert Romme. Histoire d'un révolutionnaire (1750-1795), Paris, 1971 ; J. Ehrard, ed., Gilbert Romme (1750-1795), Paris, 1996.

2. Sankt-Peterburgskij Filial Instituta Rossijskoj Istorii (SPF IRI, ex-LOII), f. 8 (carton 372), d. 96. Ce document est publié ci-dessous (Doc. I).
} 
puisque l'écriture en est identique à celle des lettres de Romme. De plus, la chemise porte la mention «Manuscrits de G. Romme », de la main du même archiviste (sans doute M. de Vissac, le premier biographe du célèbre montagnard) que des annotations analogues relevées sur d'autres papiers de Romme qui, après être restés, dans les années $70 \mathrm{du} \mathrm{XIX}^{\mathrm{e}}$ siècle, regroupés en un même fonds, sont aujourd'hui disséminés dans des archives italiennes, françaises et russes. Les spécialistes de la biographie de Romme, à tout le moins les Russes ${ }^{3}$, connaissent ce document depuis assez longtemps. Šarkova en a publié dès 1982 une description archéographique ${ }^{4}$. Il est à remarquer qu'elle manifeste un certain embarras en ce qui concerne les circonstances de la rédaction de ce mémoire : « Il est difficile de dire dans quel but Romme a rédigé ce texte un an à peine après son arrivée en Russie... » ${ }^{5}$. Mais elle ne propose aucune explication à ce fait.

Peut-être n'y a-t-il là aucun mystère? Peut-être n'avons-nous à faire qu'à l'une des manifestations de l'insatiable curiosité de Romme? En effet, si ses intérêts ont concerné les aspects les plus divers de la vie de la société russe, pourquoi «le militaire » de Russie aurait-il fait exception? D'autres voyageurs qui parcouraient l'Europe ont visité des forteresses, assisté à des revues sans arrière-pensées, gouvernés par leur seule curiosité, comme, par exemple, Casanova, qui, en 1765, a suivi pendant trois jours les manœuvres de l'armée russe.

Pourtant une étude attentive des Observations de Romme révèle que les choses sont loin d'être aussi simples dans le cas présent. D'abord, il est impossible de ne pas relever la brièveté du délai mis à leur rédaction. Romme est arrivé en Russie à la fin de l'année 1779, c'est donc en moins d'un an qu'il a mis au point une description très détaillée des forces armées russes ${ }^{6}$. Pour accomplir un travail d'une telle ampleur, et sans savoir le russe, il fallait lui consacrer beaucoup plus de temps et d'efforts qu'on n'en pourrait attendre d'un simple voyageur portant un égal intérêt à toutes les sphères de la vie d'un pays étranger. À ce propos, l'absence, dans les papiers de Romme qui nous sont connus pour 1780 , de manuscrits tant soit peu significatifs sur d'autres sujets apporte une confirmation indirecte à notre hypothèse.

D'autre part, l'aspect extérieur des Observations donne également à réfléchir. La plupart des journaux de voyage de Romme, comme ses remarques sur la géographie, la botanique et la minéralogie, sont écrits à la diable et difficiles à déchiffrer

3. Voir A. Tchoudinov, «Les papiers de Gilbert Romme aux archives russes », dans Archives historiques de la Révolution française, 304, 1996, pp. 257-265.

4. I. S. Šarkova, «Fond Gilbera Romma» (Collection Gilbert Romme), in Rukopisnye istočniki po istorii Zapadnoj Evropy v arhivah Leningradskogo otdelenija Instituta istorii SSSR (Sources manuscrites de l'histoire de l'Europe occidentale aux archives de la Section de Leningrad de l'Institut d'histoire de l'URSS), Leningrad, 1982, p. 170.

5. Ibid., p. 171.

6. La première lettre de Romme envoyée de Russie à Dubreul, son correspondent permanent, est datée du $1^{\text {er }}$ décembre (v. st.) 1779 (voir Museo del Risorgimento à Milan, fonds Romme, carton 1, dossier 11). Romme rédigea ces Observations avant le mois de novembre 1780, car Engel'gardt y apparaît en tant que colonel du régiment de hussards, or il est nommé colonel des cuirassiers le 24 novembre 1780 . 
car destinés avant tout à l'auteur lui-même. Les Observations sont écrites de l'écriture nette, parfaitement lisible dont Romme usait pour ce qu'il destinait aux yeux des autres.

Et enfin, par son contenu même, le document évoque plutôt une étude analytique que les notes d'un observateur extérieur et désœuvré. De temps à autre, on rencontre dans le texte des informations sur les efforts déployés par l'auteur pour collecter l'information de façon méthodique : «Il nous a été impossible jusqu'à présent de nous procurer encore des détails entièrement complets [...] », « il a été impossible de se procurer encore des détails sur cette cavalerie [...] », etc. Des faits sont mentionnés (par exemple, les manœuvres du régiment de dragons de Smolensk) dont Romme n'a pas été le témoin direct et dont il ne pouvait avoir connaissance qu'en ayant interrogé d'autres personnes. Il désigne même l'une de ses sources - « un officier de cavalerie qui passe pour avoir du mérite ». À certaines remarques de l'auteur, on peut encore comprendre que son étude est destinée à un lecteur averti : «on s'abstient de placer ici une nomenclature des grades du service de Russie parce qu'on les croit connus ». Bref, les Observations de Romme ressemblent beaucoup plus au rapport d'un agent secret sur l'état de l'armée russe qu'à d'anodines notes de voyageur. Voici pourquoi, après en avoir pris connaissance, j'ai été contraint de douter pour la première fois du caractère exclusivement civilisateur de l'activité de Romme en Russie.

Pourtant, la seule existence de ce document ne pouvait permettre d'affirmer que son auteur avait accompli une mission secrète, le mémoire n'avait pas été expédié : il était resté dans les archives personnelles de Romme. Le soupçon ne pouvait se transformer en certitude qu'avec la découverte de preuves irréfutables que les renseignements collectés par Romme étaient destinés au gouvernement de la France. Et puisque, dans la France de cette époque, il n'y avait pas de service centralisé de renseignements (« le secret du roi » avait cessé d'exister après la mort de Louis XV) et que c'était les services de la politique extérieure qui suivaient, en même temps que l'activité diplomatique, les activités d'espionnage, il fallait chercher les preuves de l'activité secrète de Romme dans les archives du ministère des Affaires étrangères français.

Lors de l'examen de l'inventaire du volume 14 de la série Mémoires et documents (sous-série Russie ), où se trouvent les matériaux rassemblés par les diplomates français sur l'armée russe de 1745 à 1828, le titre familier Observations sur le Militaire ne pouvait que retenir mon attention 7 . Et en effet, le manuscrit en six pages in-folio s'avéra également de la main de Romme. Bien qu'il diffère par sa forme du manuscrit pétersbourgeois, il n'en reste pas moins que, pour l'essentiel, le contenu des deux documents coïncide, des passages entiers sont même absolument

7. Paris, Archives du Ministère des Affaires étrangères (MAE), série Mémoires et documents, sous-série Russie, vol. 14, Russie 1745 à 1828 . Forces militaires, ff. 123-128vº . Ce document, publié ci-dessous (Doc. II), est déjà connu des chercheurs, mais seulement comme document anonyme. John Keep l'a cité, par exemple, en le datant, pour une raison obscure, de la fin des années 90 du XVIII siècle. Cf. J. L. H. Keep, Soldiers of the Tsar. Army and society in Russia 1462-1874, Oxford, 1985, p. 221. 
identiques. Ils sont agencés différemment dans le manuscrit parisien, ce qui en facilite l'accès, le second texte étant formalisé. Visiblement, la variante pétersbourgeoise a été rédigée un peu avant, puis a servi de base à la variante parisienne. Cette dernière est le résultat d'un travail de rédaction, de contraction et de recomposition du premier texte par l'auteur, après quoi la version améliorée a été expédiée à Paris tandis que son prototype restait dans les archives personnelles de Romme.

Le fait d'avoir établi que le gouvernement français a reçu de Romme des informations sur l'état de l'armée russe constitue en soi un témoignage convaincant de ses activités d'espionnage en Russie. Mais le dernier paragraphe du mémoire contient en outre une indication directe sur le genre de la mission qu'il assumait : « on espère, au reste de pouvoir deposer bientot aux pieds de S. M. si elle daigne l'agréer, un Tableau plus achevé dans lequel on fera entrer tous les details relatifs à la paye, à l'entretien, à l'habillement du soldat et leur evaluation, auquel on ajoutera un calcul de ce qu'un regiment de Cavallerie a couté annuellement dans l'espace d'un couple d'années consécutives... », etc.

Dans ce même quatorzième volume, les Observations sur le Militaire sont précédées d'un autre document, fort intéressant, de toute évidence d'origine analogue : il s'agit d'une note statistique intitulée État des Troupes de Russie ${ }^{8}$. Ce document y est en double exemplaire, chacun tenant sur une feuille in-folio. Le premier exemplaire (f. 121) est de la main de Romme, le second (f. 122) d'une écriture que je n'ai pu identifier. Pour le contenu, les deux exemplaires coïncident; pourtant, à en juger à quelques mots écrits en entier par Romme et abrégés pour aller plus vite dans le second exemplaire (par exemple «hommes » devenus «hom : »), ce dernier est probablement une copie qu'un scribe anonyme a faite du document dont Romme est l'auteur.

Sur les deux exemplaires de la note une date a été portée à l'encre rouge par l'archiviste, « 1779 ». N'y aurait-il pas là une contradiction? Car, nous le savons, en 1779, Romme n'a passé en Russie que la fin de l'année, il n'a donc pas pu avoir le temps de rassembler les renseignements fournis par la note. À mes yeux, cette date s'explique probablement par le fait qu'occupé en 1780 à la recherche d'informations sur l'armée russe, Romme n'a pu obtenir de données sur ses effectifs que pour l'année précédente. On ne peut, par ailleurs, exclure une erreur de la part de l'archiviste. Quoi qu'il en soit, l'écriture de Romme pour le premier exemplaire de la note, sa place dans les archives, juste à côté des Observations sur le Militaire et le contenu commun des deux documents rendent parfaitement vraisemblable l'hypothèse que Romme est également l'auteur de la note sur l'État des Troupes de Russie.

Quel est le degré de fiabilité des informations fournies par Romme? Et par conséquent dans quelle mesure peuvent-elles figurer parmi les sources d'une étude sur les forces armées russes à la fin du XVIII' siècle? Pour apporter la réponse la plus 
précise possible à ces questions, nous allons tenter d'analyser les renseignements rassemblés par Romme sous deux angles : le premier, purement militaire, et le second, social.

En ce qui concerne l'aspect militaire à proprement parler, il faut reconnaître que les rapports de Romme dressent un tableau assez éloigné de la vérité. Et ce n'est pas tant du fait de l'inexactitude des chiffres qu'il fournit, que de son appréciation négative du niveau de préparation au combat de l'armée russe. Selon Romme, les forces armées russes étaient à ce moment-là dans un état de désorganisation et de délabrement dont il rend largement responsable le prince G. A. Potemkin, viceprésident du Collège de la Guerre. En réalité, Romme, mathématicien et naturaliste, qui vient juste d'arriver dans un pays étranger, qui n'a pas encore eu le temps de se familiariser suffisamment avec ses particularités, et qui ne dispose pas de connaissances militaires approfondies, a pris pour des ruines le désordre d'un chantier.

Sous le règne de Catherine II, l'armée russe a été l'objet d'une réforme radicale et extrêmement efficace qui en a fait l'une des meilleures d'Europe pour ses qualités au combat. C'est justement durant cette période que s'élaborent et sont assimilées de nouvelles méthodes d'instruction militaire et de nouvelles tactiques. Le règlement du service dans l'infanterie adopté en 1763 laissait aux chefs d'armée russes beaucoup de liberté pour innover dans ce domaine puisqu'il instaurait des normes d'ordres de bataille obligatoires pour tous les régiments, mais laissait à la discrétion des officiers supérieurs, à partir du grade de colonel, le choix des moyens et des méthodes de préparation des régiments au combat et admettait une grande variété dans les évolutions de la troupe. C'est justement cette manifestation de l'initiative créatrice «à la base » qui a débouché sur la rédaction d'instructions aussi innovantes pour l'époque que « Usages du service » (1770) de Rumjancev, « Règlement du régiment» (1764-1765) et «La science de la victoire » (17951796) de Suvorov, « Remarques sur le service dans l'infanterie en général et chez les chasseurs à pied en particulier » (1785) de Kutuzov, etc. 9 . Or Romme porte un jugement entièrement négatif sur ces expériences limitées, les considérant comme l'une des manifestations des désaccords profonds qui se seraient emparés de l'armée russe qu'il compare même au conglomérat bariolé des nombreuses tribus formant l'armée de l'Empire germanique.

On trouve la même incompréhension chez Romme concernant les transformations introduites par le prince Potemkin dans la cavalerie. Arrêtons-nous sur la préhistoire de cette réforme. Après la guerre de Sept Ans, la cavalerie russe fut réorganisée selon le modèle occidental : à sa base, on trouvait les lourds régiments de cuirassiers et de carabiniers. Mais dans la guerre russo-turque de 1768-1774, ils ne se montrèrent pas suffisamment efficaces face à la cavalerie légère turque. Cela incita Potemkin, qui était à la tête du Collège de la Guerre en 1774, à renoncer au modèle occidental et à restructurer sa cavalerie d'une manière radicalement nouvelle. Les régiments de cuirassiers reçurent un armement plus léger et furent

9. L. G. Beskrovnij, Russkaja armija i flot $v$ XVIII veke (L'armée et la marine russes au XVIII' siècle), Moscou, 1958, pp. 385-394. 
transformés sur le modèle des régiments de carabiniers. Les effectifs de la cavalerie légère furent considérablement augmentés. Cet ensemble de mesures créa les conditions du succès de la cavalerie russe dans la guerre russo-turque de 1787$1791^{10}$.

Certes, l'appréciation négative portée par Romme sur la réforme de Potemkin peut être due dans une certaine mesure aux sources d'information auprès desquelles l'agent français puisait ses renseignements. De qui s'agit-il? Il est évident que ce ne devait pas être l'un des officiers du Collège de la Guerre; en ce cas les renseignements auraient été sans doute plus précis et plus systématiques. Il est douteux que ce fût une personne proche de Potemkin ; l'auteur du mémoire juge d'une manière partiale et injustement défavorable le prince lui-même et certains hommes de son entourage, comme V.Engel'gardt et le colonel du régiment de Narva (V. Nerančič). Au contraire, Romme apprécie F. Baur et I. Drewitz, qui ont eu de mauvaises relations avec le cercle de Potemkin. Ainsi on peut supposer que Romme obtenait ses informations auprès de militaires russes occupant une position assez élevée, qui étaient du nombre des adversaires du prince. Dans l'armée, comme à la cour - et Romme, en étant au service du comte A. S. Stroganov, avait la possibilité d'entrer en relation avec les représentants des cercles supérieurs de l'aristocratie russe, - existait une puissante opposition à Potemkin qui s'efforçait par tous les moyens de présenter ses entreprises sous un jour extrêmement défavorable. Catherine II se souviendra avec indignation de ces calomnies juste après la grandiose revue militaire du 30 avril 1787 à Kremenčug : là, elle avait enfin vu de ses propres yeux ces régiments de cavalerie légère formés par Potemkin dont on n'avait cessé de lui dire « qu'ils n'existaient que sur le papier $»^{11}$. Et c'est la vie elle-même qui se chargera de donner la réponse la plus convaincante à ces détracteurs. L'efficacité des transformations réalisées dans le domaine militaire sous le règne de Catherine par Rumjancev, Potemkin et Suvorov a été démontrée dans la pratique, à la fin du XVIIIe siècle, par les victoires remportées par la Russie.

$\mathrm{Si}$, pour ce qui est des questions à proprement parler militaires, la valeur des jugements de Romme suscite des doutes certains, ses observations concernant l'aspect social du service à l'armée en Russie, bien au contraire, sont assez précises et se trouvent confirmées par d'autres sources. C'est valable, par exemple, pour la description des méthodes de recrutement. Les abus lors de la conscription et de l'acheminement des recrues sur leur lieu de service sont un problème des plus douloureux pour l'armée russe tout au long du XVIIIe siècle. Ainsi, en 1763, le Collège de la Guerre a prié la Commission militaire chargée d'élaborer des propositions en vue de la réforme de l'armée, de déterminer au plus vite « comment dans l'avenir procéder au recrutement, comment amener les recrues, comment les

10. Ibid., pp. 316-321; C. R. Andolenko, Histoire de l'armée russe, Paris, 1967, pp. 88-89; Ch. Duffy, Russia's military way to the West. Origins and nature of Russian military power 17001800, Londres - Boston, 1981, p. 172.

11. Voir D. F. Maslovskij, Zapiski po istorii voennogo iskusstva v Rossii (Notes sur 1'histoire de l'art militaire en Russie), tome des références et suppléments, Saint-Pétersbourg, 1894, p. 26 ; V. S. Lopatin, Potemkin i Suvorov (Potemkin et Suvorov), Moscou, 1992, p. 103. 
nourrir [...], afin que, lorsqu'aurait lieu la campagne de recrutement [...], ils ne meurent pas de faim, de froid et de maladie ». Pourtant cette situation déplorable a perduré, ce dont témoigne, en particulier, le rapport daté du 15 avril 1774 du feldmaréchal Rumjancev et envoyé depuis l'armée en campagne: les recrues « épuisées par la route, amenées à marches forcées et pas à la meilleure saison, sont arrivées ici déjà dans un état de faiblesse extrême, et une grande partie d'entre eux était à l'article de la mort sans même avoir jamais été directement soumis aux rigueurs du service $»^{12}$.

Le récit de Romme sur la concussion qui fleurissait dans les régiments est parfaitement véridique. Après que les nouveaux textes, comme «Instruction à l'usage du colonel d'un régiment d'infanterie » (1764) et « Instruction à l'usage du colonel d'un régiment de cavalerie » (1766), destinés à réglementer l'administration du régiment, avaient conféré aux colonels le droit de gérer en personne et en toute indépendance l'économie des régiments qui leur étaient confiés, les abus, aux dires mêmes des contemporains, s'étaient multipliés. Voici ce qu'en écrivait le comte Semen Voroncov :

« Ce même fondateur de notre armée [Pierre Irr] sentit très bien la nécessité de séparer la partie économique de la partie qui ne regarde que la subordination et la discipline. Ces deux dernières étaient confiées au pouvoir suprême du colonel, mais la partie économique était entre les mains des délégués du commissariat [...]. Ce règlement salutaire a été enfreint par [les] règlements de 1763 , où on donna au colonel une autorité illimitée sur l'économie du régiment. Aussi, dans moins de sept ans après, j'ai été témoin des abus scandaleux que plusieurs colonels ont fait, à leur propre profit, de cette autorité qu'on leur avait donné si imprudemment. La couronne a été volée avec une impudence inconcevable, et les pauvres soldats ont été inhumainement privés du peu d'argent qui leur revenait de droit. $\gg^{13}$

Les observations de Romme sur l'avancement, sur les phénomènes de désertion et les particularités relevant du caractère national du soldat russe sont tout aussi dignes d'intérêt. Bref, sur tout ce qui ne concerne pas les questions de tactique et de structure du militaire, les rapports de l'agent français s'avèrent une source précieuse pour l'histoire de l'armée russe au XVIII ${ }^{e}$ siècle.

Ils ont été même des plus précieux, malgré toutes les inexactitudes, pour le gouvernement de Louis XVI. La correspondance politique entre Paris et l'ambassade de France en Russie témoigne qu'il était très difficile à l'époque de se procurer

12. L. G. Beskrovnij, op. cit., pp. 300, 308. Sur les nombreux mais vains projets visant à réformer le système des recrues au XVIII' siècle, voir J. L. H. Keep, op. cit., pp. 158-160.

13. S. R. Voroncov, «Zapiska grafa S. R. Voroncova o russkom vojske, predstavlennaja imperatoru Aleksandru Pavloviču v 1802 godu » (Mémoire du comte S. R. Voroncov sur l'armée russe, présenté à l'Empereur Alexandre Ire en 1802), in Arhiv knjazja Voroncova (Les Archives du prince Voroncov), Moscou, 1875, 10, pp.471-472. L'original est en français. 
des renseignements sur l'armée russe ${ }^{14}$. Il est significatif qu'aux archives du ministère des Affaires étrangères il n'y ait pas d'autres mémoires sur la réalité militaire russe, pour la période de 1769 à 1803, que celui de Romme. Mais cette activité de renseignement ne fut-elle qu'un épisode dans la vie de Romme ? On ne peut pas encore répondre définitivement à cette question. Pendant son séjour en Russie et même après son départ, Romme était dans les meilleurs termes et en correspondance permanente avec le chevalier Charrette de Colinière, officier de l'ambassade de France à Saint-Pétersbourg, responsable des services secrets. Cependant les lettres de Colinière à Romme, conservées aux Archives d'État de la Fédération Russe (GARF) à Moscou, ne contiennent aucun indice de thèmes secrets, ce qui n'est pas étonnant car la correspondance des étrangers était contrôlée par la police russe. Ainsi il faudrait effectuer de nouvelles recherches dans les archives afin de savoir si les renseignements secrets en Russie étaient pour Romme une mission permanente ou seulement l'un des épisodes d'une activité variée.

Bien des années plus tard, le comte L.F. de Ségur, ambassadeur de France en Russie de 1785 à 1787, rapportait l'une de ses conversations avec Potemkin dans ces termes :

«Il m'avait dit que l'armée russe s'élevait à deux cent trente mille hommes de troupes régulières et à trois cent mille d'irrégulières ; mais je savais par des voies assez sûres que cette armée se trouvait loin d'être complète; la discipline et l'instruction y étaient négligées ; l'indolence du prince permettait aux colonels de s'enrichir ; ceux-ci ne prenaient même pas grand soin de s'en cacher, et le chef d'un régiment de cavalerie trouvait très-naturel et très-légitime un gain annuel de vingt ou vingt-cinq mille roubles. $\gg^{15}$

La similitude entre les faits mentionnés par le diplomate français et le contenu des rapports de Romme est trop évidente pour être due au hasard. Aussi semble-t-il qu'on puisse aujourd'hui affirmer, avec une assez grande part de certitude, que nous connaissons les « voies sûres » de Ségur. Rien d'étonnant à ce qu'il ne les ait pas révélées lui-même. Il n'était guère opportun, sous la Restauration, époque à laquelle l'ancien ambassadeur rédigeait ses mémoires, de reconnaître que, dans les dernières années de l'Ancien Régime, c'était le futur jacobin et régicide, Gilbert Romme, qui recueillait des informations sur l'armée russe pour le gouvernement de la France.

Rossijskaja Akademija Nauk

(Traduit du russe par Hélène Rol-Tanguy)

Institut Vseobščej Istorii

117334 MOSCOU

Leninskij Prospekt, 32-a

e-mail :tchoudin@koi.postman.ru

14. Cf.,par exemple, la dépêche chiffrée du comte de Ségur au comte de Vergennes, le 6 mai 1785 : «Monsieur le Comte, rien n'est plus difficile que de se procurer ici des notions du militaire Russe. Le désordre et le mystère de l'administration concourent à rendre toutes les recherches aussi infructueuses que pénibles. L'ignorance de la plupart des officiers que l'on pourroit consulter achève de rebuter dans ce travail par les idées et les tableaux contradictoires et mal rédigés qu'ils présentent ». MAE, série Correspondance politique, sous-série Russie, vol. 113,ff. 276-276vº .

15. Mémoires, souvenirs et anecdotes, par M. le Comte de Ségur, Paris, 1859, t. 1, p. 379. 


\title{
DOCUMENTS*
}

\section{I \\ Observations sur le Militaire de Russie en 1780}

\begin{abstract}
Administration générale
L'administration de toutes les affaires de la guerre est confiée en Russie à un collège qui, selon l'institution, doit être composé d'un Président, d'un vice-president, de plusieurs membres de différens grades pris dans le militaire, et d'un grand nombre de subalternes ${ }^{1}$. Ce collège siège à St. Petersbourg, et n'a à Moscou qu'un comptoir qui dirige les divisions plus éloignées. L'un et l'autre s'occupent de tout ce qui à rapport au militaire, à l'exception des vivres qui sont du ressort d'une Chancellerie particulière, mais également dépendante du Président du collège de la guerre. Cette place de Président est vacante depuis la retraite du Maréchal Czernichew $^{2}$. Le Prince Potemkin vice-president en fait les fonctions, et c'est entre ses mains que tous les pouvoirs de cette branche importante se trouvent réunis ${ }^{3}$. Cependant ni lui, en qualité de ministre de la guerre, ni son collège n'ont aucune influence sur les gardes et sur l'artillerie. Celles-là dependent immediatement du Souverain, et celle-ci du Gd Maréchal de l'artillerie ${ }^{4}$. Chaque chef de division a sa chancellerie de même que chaque régiment, et ces dernières suivent l'armée. On y inscrit une copie de tous les ordres donnés par les Généraux et par les officiers du corps; et on y tient un etat de tous les rapports faits par les officiers ou bas officiers du Regiment. La moindre minutie y est déposée par écrits delà des embarras, des longueurs à l'infini. Une ordonnance de Pierre I porte de n'obeir qu'à des ordres écrits, et on a vu un jour d'affaire des Généraux refuser d'exécuter ceux que le Maréchal leur envoyoit verbalement. On prétend même que la prudence exige cette précaution
\end{abstract}

* Les trois documents qui suivent sont publiés pour la première fois. Nous avons respecté la graphie des originaux, en introduisant cependant des majuscules et en séparant les mots soudés sur le manuscrit. Le fragment du Document I, barré dans l'original, est placé entre crochets.

1. Le Collège de la Guerre, créé par un ukase de Pierre Ier en 1718, restera l'organe central d'administration des affaires militaires en Russie jusqu'en 1802.

2. Zahar Grigor'evič Černyšev (1722-1784), comte, général feld-maréchal. Il s'est distingué pendant la Guerre de Sept Ans. Après le coup d'État de 1762, il est nommé vice-président, et, en 1773, président du Collège de la Guerre, fonction qu'il quitte en 1774.

3. Grigorij Aleksandrovič Potemkin (1739-1791). De 1774 à 1784, il est vice-président du Collège de la Guerre avec droit de présidence et, à partir de 1784 , président du Collège de la Guerre et général feld-maréchal.

4. On trouve alors en Russie, à la tête de l'artillerie, un général feldzeichmeister (Grand Maître, selon Romme) qui, bien que membre du Collège de la Guerre, jouit d'une considérable autonomie. 
surtout vis-à-vis du comte Romansoff ${ }^{5}$. Depuis que le Prince Potemkin est à la tête de ce département, les affaires y sont dans une confusion difficile à imaginer. Sa lenteur et son indolence font regretter l'activité de son prédécesseur. Les ordres les plus urgens restent souvent des mois entiers dans son cabinet sans qu'il les signe, et quelquefois ils s'y perdent. Ce fut par une suite de cette négligence qu'un régiment d'infanterie se trouva égaré il y a une couple d'années sans qu'on sut à Pétersbourg ce qu'il étoit devenu. On envoya des courriers dans les provinces pour le chercher, et à la fin on apprit qu'il étoit depuis la paix de Cainardgi6 sur les frontieres du Kouban, faute de savoir la garnison qu'on lui destinoit. Il est aisé de se figurer l'etat dans lequel ce régiment dut être après un aussi étrange oubli. Comme le prince Potemkin a introduit beaucoup d'innovations dans l'armée, qu'il a surtout bouleversé l'ancien réglement de la cavalerie, et plus spécialement celui des troupes légères; il est évident que cela n'a pu qu'augmenter la confusion générale. La suite de ce mémoire en fournira plus d'une preuve.

\section{Administration particuliere des Régimens}

Chaque division a ses commissaires chargés de fournir aux régimens leur paye, leur portion de farine et de gruau, les armes et tout ce qui est nécessaire à l'habillement du soldat. Ils doivent surveiller les Colonels dans ce qui concerne l'entretien de leur régimens, et informer le collège de guerre des désordres qui pourroient s'y glisser. Au lieu de cela ils connivent avec les Colonels et concourent même à ce que ceux-ci appelent leur economie. Cette economie consiste à tirer d'un régiment tout le parti possible. Chaque Colonel regarde le sien comme un bénéfice dont il faut profiter, envisage le tems pendant lequel il le commande comme l'epoque de s'enrichir, et passe à regret au grade de Général où il n'a de revenus que ses appointemens. Détailler toutes les ruses que ces messieurs mettent en usage pour arriver à leur fin seroit un récit aussi minutieux qu'inutile ici. Il suffira d'observer que l'etat incomplet d'un régiment est une des grandes ressources de leur industrie, puisque ce n'est point au profit de la Couronne que cet état retombe, mais à l'avantage du Colonel. Aussi cherchent-ils à masquer leur déficit par une infinité de moyens. Le prétexte des maladies, celui des détachemens envoyés pour recevoir de la farine, des gruaux, du drap, objets qu'en effet ils sont dans le cas de chercher fort loin; les prétextes et une infinité d'autres souvent puériles, ne leur manquent jamais, et sont trouvés valables, soit par l'ignorance des Généraux commandans des divisions, soit par leur connivence moyennant une part au profit. C'est surtout dans un régiment

5. Petr Aleksandrovič Rumjancev-Zadunajskij (1725-1796), comte, général feld-maréchal, chef d'armée et réformateur des forces armées russes. Sous son commandement, les troupes russes remportèrent des victoires décisives sur les Turcs pendant la guerre de 1768-1774. Après la guerre, il devint général-gouverneur de la Nouvelle Russie et commandant de la cavalerie régulière de l'armée russe.

6. Il s'agit bien sûr du traité de paix de Koutchouk-Kaïnardji (1774) qui consacre les victoires russes dans la guerre russo-turque de 1768-1774. 
de cavalerie que de pareilles fraudes sont sans nombre. Chacun d'eux est censé rapporter 3000 Ducats $^{7}$ à son proprietaire, s'il entend son métier ; et on tient de la bouche même d'un Colonel de dragons que le sien lui rendoit 15 à 18000 R. par an. Aussi aucun régiment de cavalerie n'est-il complet ni en hommes, ni en chevaux. On sait de science certaine qu'entr'autres, un des carabiniers, celui de Narva actuellement en Pologne, n'a pas 200 chevaux effectifs, et peut être pas le quart de ces 200 en état de faire service ${ }^{8}$. En revanche son dernier proprietaire qui l'a eu pendant 8 ans s'est fait plus de 180000 R. de capital par ses rapines tant sur le régiment que sur les Polonnois. Parmi ceux de dragons et de piqueniers la pluspart ne fournissent qu'un echantillon de ce qu'ils devroient être.

La Couronne ne sait jamais d'avance ce que sa Cavalerie lui coutera parce que il n'y a pas de prix fixé pour les fourages. Le Colonel envoye tous les mois au Commissariat de guerre un certificat signé des magistrats de l'endroit où il est en garnison qui fixe le prix des fourrages. Ces gens pour une pinte d'eau-de-vie ou pour quelques Roubles, attestent ce qu'on veut, et la Couronne est obligée de payer le foin à 14 ou 15 cop. le $\mathrm{Pd}^{9}$, prix du marché de Pétersbourg, tandis qu'il ne coute que 4 cop. au plus dans les provinces. Des régimens qui gardent les lignes depuis le Dniepper jusqu'au Wolga, et qui peuvent avoir le foin pour rien des déserts auxquels ils avoisinent, ne se font pas moins payer celui-ci, sous mille prétextes, au même taux que les autres. La Couronne alloue à chaque cheval de cavalerie un demi-pouds ou 201 . de foin par jour; aux chevaux de dragons, piqueniers, houzards 15 1. En avoine elle destine aux chevaux des cuirassiers 4 garnitz, à ceux des carabiniers et des dragons 3, aux chevaux des houzards et des piqueniers 2 garnitz par jour. Le garnitz est un peu plus petit que la malze de Berlin ${ }^{10}$.

Tous les ouvrages qu'exige l'economie d'un régiment se font par le soldat même. Il est Boulanger, Tailleur, Cordonnier, Sellier, charron, maréchal-ferrant ;

7. Le ducat (červonec) est une monnaie d'or d'une valeur de trois roubles, frappée en Russie de 1701 à 1885 .

8. En 1780, on trouve à la tête du régiment de carabiniers de Narva un Serbe, David Nerančic ; il occupe cette fonction depuis le 8 juillet 1778. Né en 1752, il passe au service de la Russie en 1768 et prend part aux campagnes contre la Pologne (1768) et la Turquie (1769-1774) dans le régiment de hussards de Kharkov et celui des Hongrois; il s'y fait remarquer par sa bravoure. En 1777, il est nommé grand aide de camp auprès du prince Potemkin, puis officier d'ordonnance auprès de l'Impératrice. Ces fonctions entraînant de fréquents séjours à la cour, il est remplacé durant ses absences par un colonel « hors statut », le comte Cornélius de Rourke, né en 1739. Ce Livonien, entré au service de la France en 1758, se trouve au service de la Russie en 1760 et participe à la campagne de 1772 contre la Pologne. Voir Archives d'histoire militaire de l'État russe (Rossijskij Gosudarstvennyj Voenno-Istoričeskij Arhiv - RGVIA). f. 489, op. 1, d. 7149 , ff. $235-236 v^{\circ}$; d. 7164, ff. 36, 39v , 48; Stoletie voennogo ministerstva 1802-1902 (Le centenaire du ministère de la Guerre 1802-1902), Saint-Pétersbourg, t. 2, vol. 1, 1902, pp. 212, 247 (cité infra : Stoletie).

D'après les états de 1775, un régiment de carabiniers devait compter 919 chevaux d'armes. Voir Stoletie, t. 13, pt. 3, vol. 1, 1906, p. 79.

9. 1 poud $=40$ livres $=16,38 \mathrm{~kg}$.

10. Inexact. En réalité, d'après les états de 1765 , l'attribution de fourrage est la même pour tous les régiments : 15 livres de foin ( 1 livre en Russie $=0,4 \mathrm{~kg}$ ) et 3 garncy $(1$ garnec $=$ approximativement 3,3 litres) d'avoine et, en cas de manque de foin, 10 livres de foin et 4 garncy d'avoine par jour et par cheval. Voir Stoletie, t. 13, pt. 3, vol. 1, p. 75. 
bref il exerce tous les métiers dont on a besoin. Chaque régiment a son dépôt qui en campagne reste derrière l'armée, sous la garde de deux compagnies destinées à sa sureté et au travail qu'il requiert, ce qui cause encore une diminution considérable des combattans effectifs.

\section{Recrues}

Les recrues des troupes de Russie sont une espèce d'impôt que les seigneurs payent au monarque suivant le tems de guerre ou de paix et relativement au besoin qu'il a de renforcer son armée. Dans les cas ordinaires, l'Impce prend un homme sur 500 dans toute l'etendue de ses états, y compris les habitants des terres qui appartiennent à la Couronne. On suit pour les recrues le cadastre de la capitation qui doit se renouveller tous les quinze ans, et dans lequel tout mâle depuis 4 ans jusqu'à 60 est classé et enrégistré. Dans les cas urgens et inattendus tels que celui de la derniere guerre contre les Turcs, l'Impce a pris une fois un homme sur 150 et même sur $100^{11}$. D'ailleurs les Régimens n'ont point de cantons particuliers, mais on emploie par préférence les habitans de la petite Russie et du Bielgorod pour la cavalerie, et les peuples des provinces maritimes et ceux dont les habitations avoisinent les grandes rivieres et les grands lacs pour la marine.

Les abus les plus déplorables et les plus pernicieux, tant pour l'Empire en général, que pour l'armée, se sont glissés dans la manière de faire les recrues. On en charge ordinairement des subalternes employés au collège de guerre qui, dans le fond des provinces, appuyés par la cupidité des chefs résidans, ou favorisés par leur négligence exercent, en faisant ces recrues, les plus grandes vexations sur les habitans de ces contrées éloignées. L'avarice la plus criante est la regle de leur opération. Guidés par elle, leur choix ne tombe que sur ceux qui se trouvent hors d'état de se racheter, propres ou non au service. Pour economiser à leur profit les sommes destinées au transport de ces infortunés, leurs conducteurs retranchent sur leurs vivres et sur les frais du voyage tout ce qu'ils peuvent s'approprier, de sorte que couverts de haillon, ayant à peine la moitié de leur subsistance, exposés à toute les horreurs de la plus affreuse misère ces nouveaux soldats sont livrés à tous les maux que doit produire un pareil traitement. Il en resulte des maladies epidémiques très dangereuses qui, jointes au défaut de soins et de secours, font périr une foule de ces malheureux. Ceux qui peuvent s'enfuir désertent, et vont peupler les forêts de brigands; et sur un détachement de 500 hommes il en arrive à peine 100 dans les régimens auxquels ils sont destinés.

11. Pendant la guerre russo-turque, les levées s'effectuent selon les normes suivantes : en 1767, une recrue pour 300 âmes; en 1768, il y a deux campagnes de recrutement, une recrue pour 300 âmes à chaque fois; en 1769, une pour 150, ainsi qu'en 1770; en 1771, deux campagnes, une recrue pour 100 âmes; en 1772, une pour 150 et en 1773, une pour cent. Avec le retour de la paix, de 1776 à 1781, on n'a plus appelé, chaque année, qu'une recrue pour 500 âmes. Voir Stoletie, t. 1, pt. 1, vol. 1, sec. 1, pp. 121-122. 
Il n'est pas etonnant que dans un aussi vaste empire que celui de Russie, et sous un gouvernement de femme, de tels abus ne parviennent pas jusqu'au thrône ; sans cela l'intérêt du souverain autant que son humanité ne manqueroit point d'y apporter les remèdes les plus efficaces. Mais ce qu'il y a d'incompréhensible pour quiconque ignore le caractère général de la nation russe, c'est qu'aucun de ses ministres ou de ses généraux n'ait assez de patriotisme et de courage pour dévoiler au Souverain de semblables horreurs. Telle est cependant l'apathie de tous les gens en place pour le bien public, que nul ne s'en embarasse pourvu que ses propres intérêts n'en souffre pas, et ceux qui en pâtissent sont trop éloignés ou trop pusillanimes pour que leurs plaintes puissent percer. Quant aux chefs de régimens nous avons déjà allégué plus haut les raisons qui leur font dissimuler la foiblesse de leurs corps respectifs. Il est par conséquent impossible de tabler sur les états qu'on fournit des troupes russes; le vide y est compté pour le plein, et le nombre des hommes y est calculé non tel qu'il est mais tel qu'il devroit être.

Avant de terminer cet article il est essentiel de faire mention des mariages des soldats et de leurs enfans. Le paysan russe en général se marie de très bonne heure. S'il est marié et qu'il ait des enfans avant d'être soldat, ceux-ci restent eclaves du seigneur de leur pere; mais après son enrollement le seigneur n'a aucun droit ni sur sa femme ni sur ses enfans puinés dont les mâles sont soldats en naissant et reçoivent la paye. L'Impce a même établi à la suite de chaque régiment une ecole où ils apprennent à lire et à ecrire, et où ils reçoivent une sorte d'education. Parvenus à un certain age on les fait fiffres ou tambours, ou on les emploie dans le nombre incroyable d'écrivains qui composent les chancelleries militaires ${ }^{12}$.

[Quant à la paye de l'armée russe elle est en général très modique. Il nous a été impossible jusqu'à présent de nous procurer des détails entièrement complets; mais ceux que nous allons spécifier ici ont été vérifiés avec le plus grand soin. Dans l'infanterie le Grenadier a 7 R. 92 cop. de paye annuelle, le fantassin 7 R. 35. Il reçoit en outre 75 Liv. de farine par mois, environ une demi-malze de gruau, du sel et une petite portion d'eau de vie qui devient plus forte en hiver et en tems de guerre lors qu'il marche à l'ennemi].

\section{Constitution intérieure de l'armée}

Jadis le soldat russe passait individuelement pour un des meilleurs de l'Europe. Attaché non à sa patrie, mais à sa glèbe, rarement abandonnoit-il ses drapeux. Des préjugés de religion, une discipline sévère le rendoient redoutable un jour de

12. Les écoles de garnison destinées aux fils de soldats sont créées en 1721. Les élèves y apprennent à lire, à écrire, à compter, à faire l'exercice ; ils sont répartis en quatre sections selon leurs capacités : $20 \%$ y étudient « la géométrie, les sciences de l'artillerie et du génie »; $20 \%$ - les métiers de serrurier et d'armurier; $40 \%$ - le chant et la musique militaire; les $20 \%$ restants sont laissés dans les régiments pour exercer les fonctions de scribe et de sousofficier. Voir Sbornik voenno-istoričeskih materialov (Recueil de matériaux d'histoire militaire), Saint-Pétersbourg, 16, 1904, pp. 143-167 ; L. G. Beskrovnij, op. cit., p. 456 ; Ch. Duffy, op. cit., p. 130; J. L. H. Keep, op. cit., pp. 201-202. 
bataille; sa constitution physique et sa maniere de vivre lui faisoient supporter les fatigues, et surmonter les plus grands obstacles ${ }^{13}$. À bien des égards ce temps n'est plus. Les guerres du dehors lui ont appris que partout ailleurs, il peut être mieux que dans son pays natal, les prejugés disparoissent peu à peu et la désertion a commencé à se glisser dans les troupes ${ }^{14}$. D'ailleurs elles n'obéissent plus aussi auveuglément qu'autrefois, elles osent censurer les ordres de leurs superieurs et souvent refuser de les exécuter. C'est ainsi que dans les troubles de Pologne, on a vu un détachement considérable ne pas vouloir attaquer un poste sous prétexte que sa prise ne valloit pas la peine de se faire tuer. On prétend que cet exemple n'est pas unique. On auroit cependant tort d'inférer delà que le soldat soit moins rudement traité que par le passé. Les punitions anciennes subsistent dans toute leur rigueur, mais peut être les inflige-t'on souvent hors de propos. Si le soldat russe accoutumé dès le berceau à l'esclavage supporte sans murmurer les plus cruels châtimens, ce n'est, de l'aveu de tous ceux qui en connoissent le génie, qu'autant que le châtiment est juste, tandisque, maltraité sans raison, il se livre au ressentiment et à la vengeance. Or la plupart des officiers, gens sans éducation, sans principe, n'ecoutent tantôt que leur brutalité et tantôt s'abandonnent à une familiarité indécente. De tout tems l'officier russe national a moins valu que le soldat. La petite noblesse, destituée des mœurs et de connoissances, ne prend service que pour obtenir un rang et le droit de tenir carosse. Ce but obtenu elle se retire dans ses terres ou cherche quelqu'emploi et vit. Les gens à connexions entrent dans les gardes, ou comme aides-de-camp chez quelque Général et y restent jusqu'à ce que parvenus au grade d'officiers majors on les place à la tête des régimens sans qu'ils ayent la moindre idée du service. Une autre manière de se procurer de l'avancement est de passer de l'infanterie dans la cavalerie et vice-versa. On connoit beaucoup d'officiers qui dans peu d'années ont changé d'armes jusqu'à six fois afin de gagner un régiment. Une suite naturelle de l'insuffisance des officiers est le manque d'instruction du bas officier, lequel est compté au nombre des vices fondamentaux du militaire d'ici.

À tous les abus qu'on vient de détailler joignons le pouvoir des Colonels sur leur régimens. Libres d'y introduire telle nouveauté, telle lubie que bon leur semble, chaque mutation de chef ne manque jamais d'entrainer des changemens, bons ou mauvais n'importe, - d'ailleurs l'avarice leur fait, ainsi que nous l'avons déjà

13. La Commission militaire citée plus haut avait déjà relevé ces mêmes particularités de l'armée russe : «La force d'une armée ne réside pas tant dans le grand nombre de ses soldats que dans le maintien de la discipline, dans son bon entraînement, son bon entretien et sa fidélité, le plus grand fondement à tout cela étant la communauté de langue, de foi, de traditions et de race ». L. G. Beskrovnij, op. cit., p. 307. Bien des contemporains étrangers soulignèrent aussi et, selon Keep, exagérèrent même la dévotion des soldats russes. J. L. H. Keep, op. cit., pp. 204-205.

14. S. R. Voroncov lui-même témoigne des désertions massives qui touchent l'armée russe dans ses campagnes à l'étranger : «L'inhumanité avec laquelle les pauvres soldats ont été traités, les a forcés à la désertion, - chose inconnue auparavant dans les armées russes. J'ai vu des milliers de nos braves compatriotes servir dans les armées prussiennes et autrichiennes, et des personnes qui ont été en Suède m'assurent qu'elles ont vu au-delà de 2000 Russes servant dans l'armée suédoise à Stockholm et à Gothenbourg ». S. R. Voroncov, op. cit., pp. 472-473. Cependant, d'après des études récentes, les désertions furent beaucoup moins nombreuses à l'époque de Catherine II que sous les règnes de ses prédécesseurs et successeurs. J. L. H. Keep, op. cit., p. 222. 
observé plus haut, envisager leurs Régimens comme un objet de finance sans inspection que celle des commissaires complices de son manège. Le seul moment critique pour un colonel est celui où il remet le régiment et les comptes à son successeur. Mais à l'ordinaire les choses s'arrangent, et il n'y a pas d'exemple que quelqu'un n'ait été rendu responsable.

L'Etat fixe chaque régiment d'infanterie à 1886 hommes en front de bandiere ; mais ils n'y sont jamais ${ }^{15}$. Ceux qui approchent le plus du complet n'en ont pas plus de 13 à 1400 et la majeure partie est encore au dessous de ce nombre. On croit être fondé d'avancer que si une armée russe devoit à l'heure qu'il est, entrer en campagne, ce seroit beaucoup que de pouvoir compter mille combattans par régiment d'infanterie, puisqu'il faudroit deduire deux compagnies pour le dépôt. Quant au réglement de l'infanterie il est encore tel qu'on le fixa à l'avènement de l'Impce au trône; le ministre actuel de la guerre n'y a rien changé; et si le nombre des régimens à pied a été augmenté, cela s'est fait sous le ministere du Cte Czernichew ${ }^{16}$. Ce n'est que sur la cavalerie que le prince Potemkin a étendu ses vues et nous allons spécifier, autant que possible, en quoi ces changemens consistent.

Le nombre des régimens des Carabiniers a été reduit de 18 à 9, de 6 escadrons chacun. Leur réglement n'a pas été changé. Leurs chevaux plus petits que ceux des Cuirassiers ne sont taxés qu'à $30 \mathrm{R}$. pièce et le régt n'a guères au delà de $4000 \mathrm{R}$. pour la remonte annuelle ${ }^{17}$.

Tous les régimens de Dragons ont 10 escadrons y compris les 5 régimens formés de la milice d'Orembourg. Il y en a 12 en tout, aucun n'est complet. Les anciens le sont davantage que les nouveaux, mais de ceux-ci, on sait de la bouche même de leurs colonels qu'il y en a qui ne contiennent pas 400 chevaux $^{18}$; aussi un tel régiment est-il censé rapporter à son propriétaire 15 à $18000 \mathrm{R}$. et comme ces chefs sont tous gens à connexions, quoique dans leur nombre il y ait des étrangers, il n'est pas probable qu'on les oblige à compléter leurs corps. La remonte est fixée à 6000 R. et le prix du cheval à $25^{19}$. L'equipage est à la hongroise.

15. Inexact. Selon les états établis en 1763 et toujours en vigueur en 1780, un régiment d'infanterie se compose de 2093 hommes. Voir Polnoe sobranie zakonov Rossijskoj imperii s $1649 \mathrm{~g}$. (Collection complète des lois de l'Empire de Russie depuis 1649), Saint-Pétersbourg, 16, 1830, $\mathrm{n}^{\circ} 11735$. Le chiffre de 1800 fourni par Romme correspond au régiment sans les deux compagnies tenues de rester au cantonnement du régiment en tant que réserve destinée à le compléter. L. G. Beskrovnij, op. cit., p. 312.

16. Selon les états de 1763 , l'infanterie se compose de 50 régiments de campagne. À la veille de la guerre russo-turque, le nombre de ces régiments s'élève à 63. L. G. Beskrovnij, op. cit., p. 311 .

17. Inexact. D'après les états de 1765 , pour la remonte d'un régiment de carabiniers on assigne la somme de 2872,5 roubles par an. Stoletie, t. 13, pt. 3, vol. 1, p. 71.

18. Le nombre de régiments de dragons, réduit à sept en 1765, après la Guerre de Sept Ans, s'élève à dix à la fin de la guerre russo-turque, en partie du fait de la transformation des régiments de la milice. Au 1er janvier 1779, 1'armée russe compte huit régiments de dragons. Selon les états de 1775, un régiment de dragons doit compter 1565 chevaux d'armes. Voir RGVIA, f. 489, op. 1, d. 7413; L. G. Beskrovnij, op. cit., pp. 317-319; Stoletie, t. 13, pt. 3, vol. 1, p. 79.

19. Inexact. D'après les états de 1765, un régiment de dragons se voit assigner 1985 roubles pour la remonte et, pour l'achat d'un cheval de dragon, 20 roubles. Stoletie, t. 13, pt. 3, vol. 1, pp. 71-72. 
Au commencement du regne actuel la Russie n'avoit qu'une couple de régimens de Houzards réputés asses mauvais ; mais leur nombre a été augmenté considérablement pendant la derniere guerre et durant les troubles de la Pologne; et c'est peut être maintenant la meilleure cavalerie russe. Les Houzards sont de deux espèces différentes. L'une, les Houzards réguliers font un total de 7 régimens et à 6 escadrons de 135 maitres chacun sans les bas officiers. Chaque Houzard reçoit pendant la paix $12 \mathrm{R}$. de paye annuelle, en tems de guerre $15 \mathrm{R}$. par conséquent plus qu'aucun autre soldat russe. Leurs chevaux sont taxés à 15 et leur remonte à 2100 roubles $^{20}$. Ils sont assez complets, quoiqu'il n'y ait pas de régiment où il ne manque au moins 200 hommes. L'autre espèce d'houzards est appelée posselènié ou colons. C'étoit jadis une milice à cheval destinée à garder les lignes de l'Ukraine et de la Nouvelle Russie à qui on avoit donné du terrein à cultiver. Ils y établirent des villages ou slobodes et c'est de ces villages qu'on tire le fond de ces régimens posselènié depuis qu'ils ont été mis sur un pied régulier. Le plus grand nombre de ces colons est originaire de Hongrie, de Servie et de Pologne; et de ce dernier pays on en tire encore beaucoup à l'heure qu'il est. Il y a 9 de ces régimens à 6 escadrons desquels le pied de paix est de 90 et le pied de guerre de 135 hommes sans les bas officiers et chaque homme est obligé de servir 15 ans lesquels ecoulés il se retire dans son village. Outre 4 arpens de terre qu'on lui donne en propriété, il reçoit en tems de paix 8 et pendant la guerre $12 \mathrm{R}$. de paye annuelle; le reste de la composition, ainsi que la remonte des chevaux sont les mêmes que chez les autres Houzards. Le Colonel est à la tête de la colonie et doit veiller à son amélioration. Ces 9 régimens sont plus incomplets que les 7 anciens.

Les Piqueniers sont egalement posselènié. Etat, composition et remonte ne différent en rien des Houzards de ce nom. Mais, quoique sur l'état de l'armée depuis 6 ans, il s'en faut de beaucoup qu'ils soient complets. On prétend même que dans plusieurs il n'y a d'effectif que les officiers ${ }^{21}$.

Quant aux chevaux légers leur création est toute nouvelle ${ }^{22}$. C'est le Maréchal Romansow qui les forme des cosaques de l'Ukraine, les plus mauvais de tous les cosaques, dont, par cette metamorphose, on espère tirer quelque parti. Il a été impossible de se procurer encore des détails sur cette cavalerie, détails qu'on ignore

20. Inexact. D'après les états de 1765, un régiment de hussards se voit assigner 2054,25 roubles pour la remonte et, pour l'achat d'un cheval de hussard, 18 roubles. Ibid., pp. 71-72.

21. Les unités de hussards apparaissent dans l'armée régulière russe à partir de 1723; elles sont recrutées jusque dans les années 60 du XVIIIe siècle chez les Serbes, les Croates, les Hongrois, les Valaques et les Géorgiens pour assurer la garde de la frontière sud de la Russie, en qualité de milice à cheval. Vers 1763, le corps dit Novoserbskij se compose de 13 régiments de hussards. Leur tenue au combat durant la Guerre de Sept Ans avait démontré leur très faible niveau de préparation. En 1763, quatre régiments de hussards sont transformés en régiments réguliers; les autres, devenus régiments de colons (poselenie), hussards et piqueniers, assurent le service de campagne sur place. Avec la réforme décidée par Potemkin, pratiquement toutes les unités de colons seront transformées en unités régulières. Au 1er janvier 1779, on compte en effet sept régiments de hussards de campagne et neuf de hussards colons (poselenie). Voir RGVIA, f. 489, op. 1, d. 7413; D. F. Maslovskij, op. cit., pp. 28-31.

22. Les premiers régiments de cavalerie légère ont été formés en 1771. Voir D. F. Maslovskij, op. cit., p. 30 . 
même ici au collège de la guerre. On sait simplement qu'elle formera un corps de 8 régimens.

De toutes les branches du militaire russe, l'artillerie est sans contredit la plus parfaite, et celle qui s'est le mieux maintenue. Son sistème est toujours sur l'ancien pied et il s'y passe moins de désordres que dans les autres armes. Les avancemens ne se font que dans le corps. Les officiers jusqu'au Colonel inclusivement ont un grade de plus que dans le reste de l'armée. Quoique le Gd Maître de l'artillerie (Le Pce Orlow) ne s'en mêle pas beaucoup ${ }^{23}$, les Généraux qui commandent sous lui sont des gens viellis dans le métier, et plusieurs d'entr'eux passent pour avoir du mérite. Ils sont secondés par l'aptitude et le genie de la nation pour ce genre de service, le seul pour lequel elle paroisse avoir du gout et de l'application. Il n'en est pas de même de la partie du génie. À peine y trouve-t'on des gens capables d'un nivellement exact. La direction en a été confiée depuis peu au Général Baur ${ }^{24}$, et il faudra voir s'il parviendra à mettre ce corps sur un meilleur pied. Parmi les quartiers-mâtres de l'armée il y a quelques bons ingénieurs de campagne, tous etrangers; le grand nombre, est dit-on, au-dessous du médiocre.

Pour ce qui est des bataillons de garnison leur nombre est de 84 à 7 compagnies dont 5 de fantassins, une d'ouvriers et une d'invalides. La compagnie est de 100 hommes $^{25}$. Dans leur origine ces bataillons étoient destinés à être la retraite de l'officier et l'ecole du soldat; mais cette sage institution de Pierre I n'est plus suivie ; ce sont maintenant de très mauvaises troupes composée de viellards et d'infirmes. Le soldat n'a de paye annuelle que 2 R. 74 Cop., outre le pain et le gruau. Il n'est habillé de neuf que lorsqu'il ne peut plus cacher sa nudité.

\section{Manœuvres}

Les soldats russes sont lents et roides dans leurs mouvemens, très gênés dans leurs positions, ils marchent mal et n'ont jamais d'attitude aisée et naturelle. Rangés sur trois hommes de profondeur, leurs files ne sont point serrées, chaque

23. Grigorij Grigor'evič Orlov (1734-1783) occupe le poste de général feldzeichmeister (Grand Maître de l'artillerie) de 1765 à 1783; il y fait preuve de la plus grande négligence vis-àvis de ses obligations, ce qui entraîne durant cette période de sérieux abus et des dépenses excessives. Stoletie, t. 1, p. 696.

24. Friedrich Wilhelm Baur (Bauer) (1734-1783), ingénieur, général-lieutenant. Natif du comté de Hanau, il sert d'abord dans les armées de plusieurs États allemands avant de passer, en 1769, au service de la Russie. À partir de 1770 et jusqu'à sa mort, il est chef d'état-major de l'armée russe et, en 1772, la réforme de l'état-major général s'effectue selon son projet. En 1774, il part à l'étranger en raison de différends avec Potemkin. Tenu à l'écart des affaires militaires après son retour en Russie en 1775 , il se consacre à la réalisation d'importants projets d'ingénierie. Ibid., t. 7, pt. 1, 1902, p. L.

25. Inexact. D'après les états de 1764, le nombre des bataillons de garnison se monte bien à 84 mais, dès 1770, il s'élève à 92 et en 1774, à 102. Voir D. F. Maslovskij, op. cit., t. 2, p. 499. Chacun de ces bataillons se composait de six compagnies : les bataillons postés sur les frontières comportaient quatre compagnies de fantassins, une d'invalides et une d'ouvriers; les bataillons de l'intérieur en comptaient cinq de fantassins et une d'invalides. Voir Polnoe sobranie zakonov..., op. cit., t. $16, \mathrm{n}^{\circ} 12135$. 
homme ayant un espace de près de 2 pieds entre lui et son voisin. Les têtes des soldats ne sont point dirigées vers un même point de vue, la plupart ont les yeux en terre. Ils portent le fusil à bras rendurci de façon que la sous-garde repose sur l'epaule, et cette attitude fatigante les oblige très souvent à se soulager de la main droite. Généralement on semble ignorer ce que c'est qu'un alignement. Les lignes sont incertaines et flottantes pour peu que le mouvement soit rapide. Après une conversion on néglige d'aligner. Si l'assertion du Maréchal de Saxe est fondée, que ce sont les jambes qui gagnent les batailles, il sembleroit que les Russes devroient renoncer à des victoires vis-à-vis de tout ennemi discipliné. Leur feu est irrégulier, leur armes sont mauvaises. On prétend qu'au 3e coup la plupart des fusils refusent, et que les baguettes sont si mal assurées que souvent le soldat les perd. On en donne la faute à une manie qu'on a de ne point affermir les baguettes afin qu'elles fassent du bruit à la manipulation de l'arme. La manœuvre favorite de l'infanterie russe est de se former de colones en carrés et de quarrés en colonnes, et c'est celle qu'on exécute le $\operatorname{mieux}^{26}$. Il est à la vérité de jeunes Colonels qui au sortir de l'antichambre où ils ont étudié la tactique veulent essayer des manœuvres plus combinées; mais on prétend qu'elles n'aboutissent presque jamais qu'à des confusions et à n'enseigner que des balivernes. Chaque régiment et même chaque compagnie est une espèce de République où les chefs se piquent d'introduire des nouveautés sans être capables de les apprécier. Delà une disparate étonnante non seulement d'un régiment à l'autre mais entre les différentes parties d'un même corps, point d'uniformité, point de principes généraux. Aussi assure-t-on qu'une armée russe ne sauroit, à l'heure qu'il est, que former un ensemble très défectueux, et qu'elle mérite d'être comparée à une armée de l'Empire d'Allemagne.

La cavalerie est sur un pied beaucoup plus mauvais que l'infanterie. D'abord, pour ce qui est des chevaux, l'economie des Colonels les conserve jusqu'à ce qu'ils tombent de viellesse, on ne leur fournit que la moitié des fourages que le Gouvernement leur destine, se bornant à leur donner de la bouffissure, car selon un principe adopté ici, le cheval est censé bon pourvu qu'il soit gras. Par cette raison on ne les exerce que le moins possible. Pendant 9 mois de l'année on ne les sort de l'ecurie que pour les mener boire; et par conséquent la moindre fatigue les met sur les dents. Embouchure, ferrure, equipage, tout se ressent de l'industrie des Colonels. On ose parler d'autant plus affirmativement de tout cela, qu'on a vu de ses propres yeux ces désordres chez les régimens qui se relèvent ici à Pétersbourg où on ne fait venir que les meilleurs : et des officiers très instruits conviennent que chez d'autres tout est bien pire encore. Il n'y a d'exception à faire qu'en faveur du Régiment du Gd $\operatorname{Duc}^{27}$, lequel est assez bien monté et où tout est en meilleur état.

26. Dans les guerres avec la Turquie, le carré s'impose comme l'ordre de combat le plus efficace dans les opérations contre la cavalerie irrégulière, principale force de l'armée turque. Ce qui justifie l'intérêt particulier accordé, lors de la préparation du fantassin, à une formation rapide des colonnes de marche en carré et vice-versa.

27. C'est l'héritier du trône, le grand-prince Pavel Petrovič (1754-1801), futur Paul Ier (17961801), qui est le chef du régiment de cuirassiers cantonné à Saint-Pétersbourg. 
Quant aux cavaliers, il est avéré que le Russe en général, à l'exception de l'Ukrainien, n'a ni gout ni aptitude naturelle pour l'equitation ${ }^{28}$; et cet art est si peu perfectionné dans ce pays, qu'il est très difficile d'acquérir de bons principes. D'ailleurs un officier d'infanterie qui à peine saura se tenir en selle passe à la tête d'un escadron ou d'un régiment de cavalerie, et gâte par son incapacité ce que par hazard son prédécesseur peut avoir établi de sensé. Il est vrai que beaucoup de régimens ont des manèges et des ecuyers, mais ceux-ci sont pour la plus part des ignorans sans aucune teinture de l'equitation; témoin certain valet de chambre perruquier d'un ministre etranger qui se trouve actuellement ecuyer d'un régiment de Cuirassiers. La plupart des cavaliers ne savent seller ni sangler un cheval. La selle est trop en avant sur les épaules, et les sangles sont si peu assurées que pendant une manœuvre on voit quantité de chutes causées par cette négligence. Les etriers sont courts ou longs selon le bon plaisir de chaque cavalier. Après chaque exercice beaucoup de chevaux se trouvent blessés par les pieds des autres et écorchés sur le dos. Les eperons sont vissés aux talons des bottes. Or les premiers rudimens de la cavalerie étant si peu observés, il est palpable que les manœuvres s'en ressentent. Nous allons rapporter ici quelques données qui nous ont été fournies sur ce sujet par un officier de cavalerie qui passe pour avoir du mérite.

«On aime beaucoup ici les manœuvres en pleine carrière, mais comme les chevaux ne sont jamais en haleine et qu'ils n'ont pas les épaules dégourdies, ces grands mouvemens manquent de vitesse et de vigueur.

« La cavalerie est rangée en muraille à 3 hommes de profondeur. Lorsqu'après un mois de campement le grand nombre d'hommes et de chevaux malades a réduit les divisions à 6 ou 7 escouades, on fait néanmoins les quarts de conversion par divisions. Or pour gagner du terrain, ou pour favoriser le développement de la colonne, à peine le mouvement de ce quart de conversion est-il commencé qu'on commande la seconde marche.

«Pendant que le régiment marche en colonnes les officiers se trouvent à la tête des divisions, ce qui retarde la formation du régiment.

« La contre marche à 4 est entièrement abolie. Le changement de front se fait ou par une conversion entiere des divisions, ou par celle du centre. Voici ce que c'est que cette conversion du centre, un demi escadron se porte en avant à la distance de sa largeur, alors les deux demi escadrons font l'un à droite, l'autre à gauche la conversion entière. La marche oblique est entièrement ignorée. On jugera delà que doit être le développement.

« Nonobstant tout cela, on se pique de faire faire des quarts de conversion à tout le régiment, mais pour peu qu'on ait des spectateurs, on tâche toujours de choisir un

28. Voici ce qu'écrit S. R. Voroncov à ce propos : «En parlant de ce caractère distinctif des deux principales nations qui composent l'Empire Russe, quoique de même origine, mais ayant des mœurs et des habitudes aussi différentes qu'est le dialecte qu'ils ont, quoique leur langue dérive de la même source, il est à remarquer que si ceux de la Petite Russie [1'Ukraine] sont incomparablement meilleurs cavaliers que ceux de la Grande, ceux-ci sont sans contredit les meilleurs fantassins de l'univers [...]. » S. R. Voroncov, op. cit., p. 480. 
champ qui fournisse beaucoup de poussiere afin de pouvoir par là masquer les désordres ».

Suivant le nouveau réglement ${ }^{29}$, les Dragons doivent être exercés plus qu'auparavant aux manœuvres de cavalerie. À en juger cependant d'après celles qu'on a vu exécuter ici l'an dernier par le régiment de Smolensk, qui passe pour un des meilleurs, leur progrès font très peu de chose ${ }^{30}$. À peine savent-ils se tenir à cheval. D'ailleurs leurs chevaux ne sont pas en proportion de la taille assez haute de leurs cavaliers. Les seuls sept anciens régimens de Houzards ont la réputation d'être bons; mais il est à craindre qu'ils ne se maintiennent pas dans cet état vu les colonels qu'on leur donne, la plus part jeunes gens, qui n'ont nulle idée du service.

\section{Troûppes irrégulières}

La Russie peut mettre en campagne un essaim énorme de troupes irrégulières. On evalue leur nombre à près de 300000 hommes qui, pendant la paix, ne lui coutent rien à entretenir. En tems de guerre leur paye est différente; les Cosaques Tschugujew ou Cosaques Rouges, reçoivent $30 \mathrm{R}$. de paye annuelle par tête; ils se fournissent d'habits, d'armes et amenent 4 chevaux avec eux pour lesquels la Couronne leur fournit le fourages Les Cosaques du Jaïk, du Don, de l'Ukraine, ainsi que les Baschkires, et ce qui existe encore de Kalmouks n'amènent que 2 chevaux chacun et ne reçoivent de solde que $12 \mathrm{R}$. Toutes ces troupes sont divisées en régimens de 500 hommes. Les Cosaques Tschugujew et du Don sont les meilleurs, tandis que ceux de l'Ukraine ne valent rien du tout. Et voilà pourquoi l'on cherche maintenant à les mettre sur un pied discipliné. Reste à savoir si on réussira à en faire quelque chose ${ }^{31}$.

\section{Grades militaires en Russie}

Pierre I ayant lui-même mis en usage cet axiome, que pour savoir bien commander il faut savoir bien servir; voulut que tous les sujets destinés au service

29. Le nouveau « Règlement militaire sur l'exercice à cheval » a été adopté en 1763. Polnoe sobranie zakonov..., op. cit., t. 16, suppl. 2.

30. Le régiment de dragons Smolenskij est cantonné dans le gouvernement de la Nouvelle Russie. C'est pourquoi Romme, qui réside à Saint-Pétersbourg, ne peut juger de ses manœuvres que d'après le témoignage de tiers.

31. Les réformes de G. A. Potemkin et P. A. Rumjancev, entreprises à la fin de la guerre russoturque de 1768-1774, modifient considérablement l'organisation des troupes cosaques et élèvent sensiblement leur aptitude au combat. Sept régiments - trois de Čuguev, deux du Caucase et deux d'Orenburg, deviennent des troupes régulières ; on rapproche les troupes du Don de l'organisation régulière. Après la liquidation de la Seč' des Zaporogues en 1775, on commence à former de nouvelles unités avec les anciens Zaporogues, ce qui est parachevé en 1788 , avec la création de la force cosaque de la mer Noire, dite plus tard du Kouban. Au 1er janvier 1779, les effectifs des unités irrégulières de l'armée russe représentent 48801 hommes. Voir RGVIA, f. 489, op. 1, d. 7413; D. F. Maslovskij, op. cit., t. 2, pp. 68-72. 
militaire n'y pussent entrer que comme soldat, et que successivement ils parcourussent tous les grades du service avant de parvenir aux emplois supérieurs. Cette loi est encore en vigueur et un Feld-Maréchal des armées de Russie a été soldat, caporal, sergent. Mais comme le service de bas officier a paru trop dur aux gens de condition, et que les galons qui servent à désigner ce grade sont chargés ici d'un avilissement aussi injuste que déplacé, les familles puissantes ou à connexion ont voulu eviter à leurs enfans la peine d'être bas officiers. Pour cet effet, on les fait entrer dans les gardes ou ailleurs presqu'au sortir du berceau; de sorte qu'on voit une foule de caporaux ou de sergens de 7 ou 9 ans qui font le service dans leur enfance et se poussent insensiblement sans quitter la maison paternelle ${ }^{32}$. Ils se trouvent officiers sans avoir acquis la moindre connoissance du métier des armes et apportent aux régimens une ignorance totale dont ils ne sortent presque jamais. Les gens, au contraire, que la misère, la disgrace ou d'autre circonstances ecartent du thrône, n'entrent comme bas officiers qu'à l'age prescrit, s'avancent difficilement, et croupissent longtems dans la fange des corps de garde avant de devenir officiers. Ceux-ci connoissent peut être mieux la discipline et le service, mais ils contractent dans leur premier etat les vices crapuleux des soldats et des bas officiers russes et rarement ils deviennent bons militaires.

On s'abstient de placer ici une nomenclature des différents grades du service de Russie parce qu'on les croit connus. On observe seulement qu'il y a dans celui de Major une division en premier et en second Major; et qu'entre le Colonel et le Général il existe le grade intermédiaire de Brigadier ${ }^{33}$.

Comme tous les arrangemens de Pierre I sont calqués sur le militaire, l'etat civil range avec l'armée. Il en résulte quelque fois du ridicule, souvent même des abus. Cependant sous ce regne-cy il y a moins de l'un, et l'on veille davantage aux autres que du tems d'Elisabeth, où chaque laquais de la Cour étoit capitaine et pouvoit passer comme tel dans l'armée et où la gouvernante des Demoiselles de l'Impce ainsi que l'Intendant des vaches hollandoises que cette princesse se plaisoit à entretenir, avoient chacune le rang de Colonel.

Depuis l'année 1775 on peut reconnoître le grade ou le rang des personnes au nombre des chevaux attelés à leurs voitures. Les bas officiers n'ont qu'un cheval à un traineau ou à une calêche; les officiers jusqu'au capitaine inclusivement vont à 2 chevaux; depuis le Major jusqu'au Colonel à 4; depuis le Brigadier jusqu'au Lieutenant Général à 6 , et les deux premieres classes ont outre ces 6 chevaux, deux estaffiers à cheval qui les précédent.

32. Les chiffres cités ci-dessous nous renseignent sur l'ampleur de la pratique, pour les jeunes nobles, du service nominal dans les grades inférieurs : en 1792, pour un effectif officiel de 80 sous-officiers par régiment, au Preobraženskij, on en compte 6 134, au Semenovskij - 654, à l'Izmajlovskij - 1834 . En se faisant verser dans l'armée, le sous-officier de la garde reçoit le grade d'officier. C'est ainsi que sont complétées jusqu'à un tiers des vacances d'officiers dans l'armée. D. F. Maslovskij, op. cit., tome des références et suppléments, p. 7.

33. Second major est le grade d'officier qui suit celui de capitaine ; introduit en 1716, il est supprimé en 1797. Créé en 1711, le grade de premier major, qui vient juste après, est supprimé en 1797. Le grade de brigadier vient après celui de colonel ; créé en 1722, il disparaît en 1799. 


\section{Observations sur le Militaire}

Si le gouvernement de l'Empire de Russie n'offre pas un aspect avantageux le militaire y est peut être dans un plus grand désordre encore. Destitué de connoissances propres à approfondir une matiere aussi compliquée et aussi difficile que c'est un systeme militaire on se contentera ici de rapporter des faits averés et on ne se permettra que d'y joindre l'opinion des gens du metier qu'on croit les plus capables d'en porter un jugement solide.

Jadis le soldat russe passait individuelement pour un des meilleurs de l'Europe. Attaché non à sa patrie mais à sa glèbe, rarement abandonnoit-il ses drapeux. Des préjugés de religion, une discipline severe le rendoient redoutable un jour de bataille; sa constitution physique et sa maniere de vivre lui faisoient supporter les fatigues, et vaincre les plus grands obstacles. Ce temps n'est plus. Les guerres du dehors lui ont appris que partout ailleurs, il peut être mieux que dans son pays natal, les prejugés disparoissent peu à peu et la désertion a commencé à se mettre dans les troupes. D'ailleurs elles n'obeissent plus aussi auveuglement qu'autrefois, osent censurer les ordres de leurs superieurs et souvent refuser de les executer. C'est ainsi que dans les troubles de Pologne, on a vu un detachement considerable ne pas vouloir attaquer un poste sous pretexte que sa prise ne valoit pas la peine de se faire tuer, et on pretend que cet exemple n'est pas unique. Le relachement dans la discipline est sans doute la premiere cause de ce mal, non que le soldat soit moins rudement traité que par le passé, mais parce qu'on lui inflige des punitions hors de propos et que l'ignorance des officiers les fait mepriser de leurs subalternes. Au nombre des vices fondamentaux du militaire d'ici, on compte le manque d'instruction du bas officier, qui n'est qu'une suite naturelle de l'insuffisance des officiers depuis l'Enseigne jusqu' au General. Il est connu que l'officier russe vaut beaucoup moins que le soldat : la petite noblesse sans education, sans principes quelconque ne prend service que pour avoir un rang et le droit de tenir carosse. Ce but obtenu elle va vivre dans ses terres, ou cherche quelqu'emploi civil. Les gens à connexions entrent dans les Gardes, ou comme aides de camp chez quelque General, et y restent jusqu'à ce qu'ils soient parvenus au grade d'officier majors. Alors on les place à la tete des regiments sans qu'ils ayent la moindre idée du service. Une autre maniere de se procurer de l'avancement est de passer de l'Infanterie dans la Cavalerie et vice versa. On connoit beaucoup d'officiers qui dans peu d'annés, ont changé d'arme jusqu'à 6 fois, afin de gagner un regiment. Les desordres qui resultent de pareils abus sont palpables, mais ce ne sont pas les seuls qui deteriorent l'Armée russe. Mais avant d'entrer dans des details à ce sujet, on se propose de parler de chaque arme separement, et d'esquisser autant qu'il sera possible l'etat ou elle se trouve.

L'artillerie a ete de tous temps la branche du militaire russe, la plus parfaite, et c'est encore celle qui s'est le mieux maintenue. Son systeme est toujours sur l'ancien pied. Il s'y passe moins de desordres que dans les autres, et les avancements ne se font que dans le corps. Quoique le Grand Maitre de l'Artillerie, le 
prince Orloff ne s'en mele pas beaucoup, les Generaux qui commandent sous lui sont des gens vieillis dans le metier et plusieurs d'entre eux passent pour avoir du mérite.

La partie du Genie au contraire est absolument négligée; à peine y trouveroit on des gens capables d'un nivellement juste et exact. L'etat general sous la direction du General Bauer fournit quelques bons ingenieurs de Campagne la plupart etrangers, mais le grand nombre de l'aveu meme du Chef est au dessous du mauvais.

L'Infanterie, outre les defauts, tant du soldat que de l'officier qui commencent à se glisser dans l'armée, et dont on a deja fait mention, est à ce que pretend, moins bonne qu'elle ne l'etoit autrefois, quoique son reglement soit le meme, et que le Ministre actuel de la Guerre n'y ait rien changé. Le mal doit être que les Colonels faute d'une inspection stricte, introduisent dans leurs regiments telle nouveauté qui leur plait, et que chaque mutation ne manque pas d'en entrainer, bonne ou mauvaise n'importe. On accuse d'ailleurs des jeunes gens et des courtisans avancés par la faveur, qui pour se desennuyer dans les antichambres, où ils ont passé leur vie avant d'avoir un commandement, ont lu quelques livres de tactique, pour se desennuyer de vouloir essayer de grandes manoeuvres qui n'aboutissent qu'à de la confusion, font oublier ce qu'on savoit et n'enseignent que des balivernes. On ajoute que les regiments n'etant point exercés d'après des principes uniformes ne sauroient reunis en corps former qu'un ensemble defectueux, et qu'une Armée russe à l'heure qu'il est, ressemble à une Armée de l'Empire en Allemagne. Quant à la constitution interieure de l'infanterie, elle n'est pas moins delabrée que la tactique en est vicieuse. L'etat fixe chaque regiment à 1800 hommes en front de bandiere, mais ils n'y sont jamais. Ceux qui approchent le plus du complet, n'en ont pas plus de 13 à 1400 et la majeure partie est au dessous de ce nombre. On croit etre fondé d'avancer que si une Armée russe devoit à l'heure qu'il est entrer en Campagne, ce seroit beaucoup que de pouvoir compter mille hommes par regiment d'Infanterie, car il faudroit toujours en deduire 2 compagnies qui restent au depot, tant pour le garder que pour travailler aux differents ouvrages qu'exige son economie, puisque c'est par le soldat meme que se font les pieces qui composent son habillement et son equipage. $\mathrm{Au}$ reste ce n'est point au profit de la Couronne que retombe cet etat incomplet de l'Armée, le Colonel seul en retire de l'avantage, et voila pourquoi ces Messieurs ne demandent pas mieux que d'avoir peu de monde et qu'ils deguisent autant que possible la force de leurs regiments. Chaque Colonel ne regarde le sien, que comme un benefice dont il faut profiter, envisage le temps pendant lequel il le commande, comme l'epoque de s'enrichir, et passe à regret au grade de General, où il n'a de revenue que ses appointements. De pareilles fraudes restent impunies, parce que les Generaux des divisions n'examinent presque jamais avec soin les regiments, qu'ils n'y entendent rien, ou qu'ils connivent avec les Colonels moyennant une part au profit, et que d'ailleurs ces derniers peuvent masquer leur deficit, sous pretextes de maladies, de detachements en voye pour recevoir de la farine, du gruau, du drap etc. objets qu'en effet ils sont souvent obligés de chercher fort loin. Ces raisons sont toujours trouvée valables, et on les employe. Le seul moment critique pour un Colonel, est celui où il remet le regiment et les comptes à son successeur. Mais à 
l'ordinaire la chose s'arrange, et il n'y a pas d'exemple que quelqu'un ait été rendu responsable.

La Cavalerie a subi beaucoup de changements sous le Ministere du Prince Potemkin. On va specifier, autant que faire se pourra, en quoi ils consistent.

Des 5 regiments de Curassiers, qui se trouvent dans l'armée, il n'y a que celui du Gd. Duc sur l'ancien pied, les autres quatre ont perdu les cuirasses ${ }^{34}$, et l'equipage de cheval qu'ils avoient auparavant, et on leur a donné des selles à la houzarde, des bottes plus legeres, et un sabre courbé vers la pointe. Leur chevaux doivent être au moins de 5 pieds trois pouces, la Couronne bonnifie $60 \mathrm{R}$. par cheval et fixe $8300 \mathrm{R}$. annuellement pour la remonte de chaque regiment ${ }^{35}$.

Les Carabiniers composes aussi de 6 escad. sont tels qu'ils etoient autrefois. On n'a rien changé à leurs reglements. Leurs chevaux plus petits que ceux des Curassiers, ne sont taxés qu'a $30 \mathrm{R}$. et le regiment n'a guere au dela de $4 / \mathrm{m}$ R. pour la remonte annuelle.

Tous ceux de Dragons ont 10 Escadrons. On pretend que selon la nouvelle ordonnance, ils doivent etre exercés plus qu'auparavant aux evolutions de Cavalerie, mais à en juger par celles qu'on a vu executer l'ete dernier, par le regiment de Smolenski, qui passe pour l'un des meilleurs, leur savoir faire est peu de chose. Bien plus, c'est qu'ils ne savent pas meme se tenir à cheval. Les chevaux des Dragons sont trop petits, et nullement proportionnés à la taille assés haute des cavaliers. L'equipage est à la hongroise. Au reste, la plus part des regiments de Dragons n'existe que sur le papier, ou ne fournit simplement qu'un echantillon de ce qu'il devroit etre. Aucun n'est complet. Les anciens le sont plus que les nouveaux, mais de ceux ci, on sait de la bouche meme de leurs Colonels, qu'il y en a, qui ne contiennent pas 400 cheveaux. Aussi un tel regiment est-il censé rapporter à son chef 15 à $18 / \mathrm{m}$ R. et comme ces chefs sont tous gens à connexion quoique dans leur nombre, il y ait des etrangers, il n'est pas probable qu'on les oblige à augmenter le nombre des hommes dans leurs regiments. La remonte est fixée à $6 / \mathrm{m} \mathrm{R}$. et le prix du cheval à 25.

Les Houzards sont d'institution differente. Les anciens regiments ont 6 escadrons et ceux-ci 135 Maitres sans les bas officiers. Chaque houzard a $12 \mathrm{R}$. de paye annuelle tandis que les autres cavaliers ne reçoivent que 7 R. 42 kop. Leurs chevaux sont taxés à 15 et leur remonte à $2100 \mathrm{R}$. C'est la Cavalerie qui approche le plus du complet quoiqu'il n'y ait pas de Regiment où il ne manque au moins 200 hommes, et on pretend qu'ils sont bons. Tout ce qui est à craindre, c'est qu'ils ne se maintiennent pas sur ce pied, vu les Colonels qu'on commence à leur donner. Le regiment de

34. Le feld-maréchal P. A. Rumjancev est à l'origine du renoncement à la cuirasse. Selon le témoignage du comte Voroncov, « il était intimement convaincu que les cuirassiers sont moins utiles qu'une cavalerie plus légère, et toute l'armée russe sait que, dès qu'il a pris le commandement, il ôta les cuirasses [...]. » S. R. Voroncov, op. cit., p. 483.

35. Inexact. D'après les états de 1765 , on assigne 5745 roubles par an pour la remonte d'un régiment de cuirassiers. Stoletie, t. 13, pt. 3, vol. 1, p. 71 . 
Drevits $^{36}$ a un jeune homme de 22 ans, neveu du Prince Potemkin, et aide de camp de l'Imperatrice, nomme Engelgard ${ }^{37}$, qui n'a jamais servi, et plusieurs autres ont eu le meme sort.

Outre ces huzards, il y a 9 regiments appellés Houzards posselenié ou colons. C'étoit jadis une milice à cheval destinée à garder les lignes de l'Ukraine et de la Nouvelle Russie, à qui on avoit donné des terreins à cultiver. Ils y etablirent des villages ou slobodes, et c'est de ces villages qu'on tire maintenant les fonds des régiments posselenié, depuis qu'ils ont été mis sur un pied regulier. Le plus grand nombre de ces colons est originaire de Hongrie, de Servie ou de Pologne, et de ce dernier pays, on en tire encore beaucoup à l'heure qu'il est. Chaque regiment a 6 escadrons, dont le pied de paix est 90 et le pied de guerre 135 hommes, sans les bas officiers, et chaque homme est obligé de servir 15 ans, lesquels ecoulés, il se retire dans son village. Outre 4 arpens de terre qu'on lui donne en proprieté, il reçoit en temps de paix 8 et pendant la guerre $12 \mathrm{R}$. de paye par an. Le reste de la composition, ainsi que la remonte du Regiment sont telles que chez les autres Houzards. D'ailleurs le Colonel est à la tete de la colonie, et doit veiller à son amelioration. Ils sont plus incomplets que les autres Houzards, et moins bons.

Les Piqueniers sont egalement Poselenié. Etat, composition et remonte ne different en rien des Houzards de ce nom. Mais quoique sur l'etat de l'armée, depuis 5 ans, il s'en faut de beaucoup qu'ils soient complets, on pretend meme que dans plusieurs il n'y a d'effectif que les officiers.

Quant aux cheveaux legers, comme leur création est toute nouvelle, on n'a pu encore se procurer des notions à leur sujet. Tout ce qu'on sait, c'est qu'ils seront formés des Cosaques de l'Ukraine, les plus mauvais de tous les Cosaques, des quels par cette metamorphose, on espere à l'avenir tirer quelque part.

36. Ivan Petrovič Drewiz (1733-1783), colonel. Il passe en 1759 du service de la Prusse à celui de la Russie. Il commande le régiment de hussards Serbskij et, de 1768 à 1772, pendant la guerre contre la Confédération du Bar en Pologne, il se fait connaître comme un chef militaire médiocre, qui se distingue avant tout par sa cruauté et le pillage de la population civile, ce que condamnera Suvorov. Il participe ensuite à l'écrasement de la révolte de Pugačev. Fait général brigadier le 22 septembre 1775, il est nommé général major le 28 juin 1777. Voir RGVIA, f. 489, op. 1, d. 7164, f. 20; A. V. Suvorov, Pis'ma (Correspondance), Moscou, 1986, pp. 1416.

37. Vasilij Vasilevič Engel'gardt (1750 - après 1800), neveu de Potemkin. Son nom figure dans les états de service dans l'armée à partir de 1766 : en 1769, il est enseigne, en 1770 sous-lieutenant, en 1772 lieutenant et, sur un ukase nominal du 15 juillet 1774, sous-lieutenant de la garde. Pendant la guerre russo-turque de 1768-1774, il participe au siège de Silistrie (1773). Le 22 septembre 1775, il est nommé aide de camp du comte P. A. Rumjancev. En décembre 1775, il reçoit le grade de colonel et accède à la fonction de commandant du régiment des hussards de Biélorussie. Il sera à partir de 1777 officier d'ordonnance auprès de 1'Impératrice. (Voir RGVIA, f. 489, op. 1, d. 7149, ff. 907vㅇ-908; d. 7152, ff. 755vº-756; Stoletie, t. 1, 1906, p. 89). Comme on peut le voir, en dépit de l'affirmation de Romme, Engel'gardt a bien une expérience du service armé puisque, avant d'être nommé officier d'ordonnance, il occupe la fonction de commandant. Sa carrière militaire se poursuivra avec succès : il recevra, le 24 novembre 1780 , le grade de brigadier et exercera la fonction de commandant du régiment impérial de cuirassiers; en 1783, il passera major général, en 1790 lieutenant général. Reversé dans le service civil sous le règne de Paul Irr, il est mis à la retraite en 1800. Voir RGVIA, f. 489, op. 1, d. 7164, f. 35v ${ }^{\circ}$; Catherine II et G. A. Potemkin, Ličnaja perepiska, 1769-1791 (Correspondance privée, 1769-1791), Moscou, 1997, p. 894. 
La Cavalerie russe, en general est bien inferieure à l'Infanterie. Le Russe, à l'exeption de l'Ukrainien, n'a aucune aptitude naturelle pour l'equitation, et cet art est si peu perfectionné dans ce pays, qu'il est impossible d'acquerir des bons principes. D'ailleurs un officier d'infanterie qui à peine saura se tenir en selle passe à la tete d'un escadron ou d'un regiment de Cavalerie, et y introduit tel usage, telle lubie que bon lui semble, et gate ce que, par hazard, son predecesseur peut y avoir etabli de sensé. Nulle regle generale, nulle uniformité, non seulement d'un regiment à l'autre, mais meme dans les escadrons. Ceux les premiers qu'on cite, comme des exemples, tels que celui de Novotroizky, dont le prince P. est chef ${ }^{38}$, savent tout au plus faire une attaque passable sur un gazon uni, mais dès qu'il s'agit seulement de quelque conversion, la confusion ne manque jamais. On excepte le seul regiment du Gd. Duc, qui outre cela est fort bien monté. Dans tous les autres, les chevaux sont très mauvais, et il n'y a de difference que du plus ou du moins. Les Colonels pour faire ce qu'ils appellent leur economie, conservent les cheveaux jusqu'à ce qu'ils tombent de vieillesse. Ils ne leur fournissent que la moitié des fourages que le Gouvernement leur destine, et se bornent à leur donner de la bouffissure, car dès que le cheval est gros il est censé bon. Par cette raison, on ne les exerce que le moins possible, pendant 9 mois de l'année on se contente de les promener tous les jours un quart d'heure au pas, et par conséquent la moindre fatigue les met sur les dents. Embouchure, ferrure, equipage, tout est generalement pitoyable. On ose parler d'autant plus affirmativement sur tout cela, qu'on a vu de ses propres yeux ces desordres chés des regiments qui se relevent ici à Petersbg. où on ne fait venir que les meilleurs, et des officiers instruits conviennent que chés d'autres, tout est bien plus mauvais encore. C'est par cette voye qu'on sait qu'un des Régiments des Carabiniers actuellement en Pologne, celui de Narva n'a pas 200 chevaux effectifs et peut etre pas le quart de ce nombre capable de faire service. En revanche son dernier Colonel qui l'a eu pendant huit ans, s'est fait plus de 180/m R. de bien, par ses rapines, tant sur le Regiment que sur les Polonois. Aussi un regiment de Cavallerie est il estimé rapporter à son proprietaire jusqu'à 7/m Ducats, s'il entend son metier. Ceux qui se contentent de 4 à $5 / \mathrm{m}$ passent pour des très honnetes gens. Un Colonel a de droit mille Roubles d'appointements. La Couronne ne sait jamais d'avance ce que sa Cavalerie lui coutera parce que il n'y a pas de prix fixé pour les fourrages. Le Colonel envoye tous les mois au Commissariat de guerre un certificat signé des magistrats de l'endroit où il est en garnison qui fixe le prix du fourrage. Ces gens pour une pinte d'eau-de-vie ou pour quelques Roubles, attestent ce qu'on veut, et la Couronne est obligée de payer le foin à 14 ou 15 cop. le poud, tandis qu'il ne coute que 4 au plus. Dans les provinces des regiments qui gardent les lignes du Cuban, et qui peuvent avoir le foin pour rien des deserts aux quels ils avoisinent, ne se font pas moins payer celui ci sous mille pretextes au meme taux que les autres. La Couronne alloue à chaque cheval 1 demi poud ou 20 livres de foin par jour, aux chevaux de Dragons, Huzards et Piquinier 15 1. En avoine elle destine aux chevaux

38. G. A. Potemkin est devenu le chef du régiment de cuirassiers Novotroickij en 1775. Voir Sbornik voenno-istoričeskih materialov, op. cit., 6, 1893, p. 44. 
des Curassiers 4 garnitz, à ceux des Carabiniers et des Dragons 3, aux chevaux de Houzards et de Piqueniers 2 garnitz par jour; le garnitz est un peu plus petit que le metre de Berlin.

Les Bataillons de Garnison, étoient designé dans leur origine à étre la retraite de l'Officier et l'Ecole du Soldat. Cette sage institution de Pierre 1er n'est plus suivie. La plus part de ces Bataillons n'est composée que de vieillard ou d'infirmes, et ce sont sans contredit de très mauvaises troupes. Chaque regiment n'a que 7 compagnies, dont 5 de fantassins, une d'ouvriers et une d'invalides. La Compagnie est de 100 hommes. Le soldat n'a de paye annuelle que 2 R. 74 cop. outre le pain et le gruau. Il n'est habillé de neuf que lors qu'il ne peut plus cacher sa nudité.

Telle est l'esquisse qu'on est à même de presenter dans ce moment ci de l'Armée Russe. On sent que cette esquisse est imparfaite, mais au moins est elle calquée sur les traits les plus saillants. On espere, au reste de pouvoir deposer bientot aux pieds de S.M. si elle daigne l'agréer, un Tableau plus achevé dans lequel on fera entrer tous les details relatifs à la paye, à l'entretien, à l'habillement du soldat et leur evaluation, auquel on ajoutera un calcul de ce qu'un regiment de Cavallerie a couté annuellement dans l'espace d'un couple d'années consécutives afin de pouvoir estimer avec probabilité le total de la somme que le Militaire russe coute à Sa Souveraine.

\section{III}

\section{État des Troupes de Russie}

La Russie a cinq Régiments d'Artillerie de Campagne

à 2000 hommes chacun, fait

10.000 hommes ${ }^{39}$

Trois regiments de Garde à pied formant

6.300

Un de Garde à cheval de

750

de Sorte qu'en y ajoutant d'Infanterie

143.10040

et de Cavalerie

$57.394^{41}$

le Total de Son Armée de Campagne sera de

217.544

39. Selon les états de 1763, l'artillerie de campagne se compose d'un régiment de bombardiers (2 295 hommes), de deux régiments de canonniers (2 392 hommes chacun) et de deux régiments de fusilliers (2 230 hommes chacun), ce qui fait au total 11578 hommes. Polnoe sobranie zakonov..., op. cit., t. 16, $\mathrm{n}^{\circ} 11784,11797$.

40. Les données de Romme concernant l'infanterie semblent considérablement minimisées. En 1774, elle compte déjà 180879 hommes, régiments de la Garde compris et, par la suite, elle comptera jusqu'à 218386 hommes en 1786. Voir L. G. Beskrovnij, op. cit., pp. 313-314.

41. Les données de Romme concernant la cavalerie sont proches des chiffres officiels dont nous disposons : 54452 hommes en 1774, 62416 hommes et 51331 chevaux d'armes en 1786. Voir ibid.,p. 319. 
Mais afin de pouvoir evaluer les Armées, qu'en cas de guerre imprévue, elle seroit en etat de faire agir, il est a considerer

$1^{\circ}$ Que presentement un Regiment d'Infanterie, l'un portant l'autre, n'a pas plus de 1200 hommes, desquels il faut deduire 2 compagnies qui resteroient au depot, circonstances qui feroient pour l'Infanterie une diminution de $62.000 \mathrm{~h}^{42}$ $2^{\circ}$ Que vu l'Etat incomplet de la Cavallerie, et les detachements pour les depots, ce n'est pas trop que de retrancher de celle-ci un tiers, ou

sont à peu de chose près indispensables, pour le maintien de la tranquillité dans ces provinces, et ne peuvent en sortir.

$5^{\circ}$ Enfin, que les Gardes depuis 40 ans ne vont pas à la guerre et n'y seroient d'aucun usage, ce qui cause encore une diminution de

Or toutes ces sommes reunies font un total de 111.760 hes

Il en resulte que dans ce moment la Russie pourroit tout au plus, mettre en Campagne, une Armée regulière de

105.784 hes

42. Pour effectuer ses calculs, Romme part des états de 1763, selon lesquels, en temps de guerre, deux compagnies doivent toujours rester au cantonnement. Pourtant, en pratique, pendant la guerre de 1768-1774, l'intégralité des effectifs des régiments, y compris les compagnies affectées à la réserve, est engagée dans les combats. Voir ibid., p. 312. 